\title{
BIFURCAÇÃO E CAOS EM CIRCUITOS ELETRÔNICOS: SIMULAÇÃO E EXPERIMENTO
}

\author{
Alípio Monteiro Barbosa*, Samir Angelo Milani Martins ${ }^{\dagger}$ \\ *Programa de Pós-Graduação em Engenharia Elétrica, Universidade Federal de Minas Gerais \\ Av. Antônio Carlos 6627, 31270-901 \\ Belo Horizonte, $M G$, Brasil \\ ${ }^{\dagger}$ GCoM - Grupo de Controle e Modelagem, Departamento de Engenharia Elétrica \\ Universidade Federal de São João del-Rei, Praça Frei Orlando 170 - Centro, 36307-352 \\ São João del-Rei, Minas Gerais, Brasil
}

Emails: alipiomonteiro@yahoo.com.br, martins@ufsj.edu.br

\begin{abstract}
This paper presents numerical and experimental studies, evidence of bifurcation and chaos in electronic systems by means of numerical and experimental analyzes. Bifurcation and chaos are observed in electronic circuits as simple as a $R L D$ (resistor-inductor-diode) circuit, an electronic system that emulates the Duffing's system and an electronic DC-DC Buck converter. For the DC-DC Buck converter, it was verified the presence of chaotic behavior into the numerical and experimental analysis, equivalently.
\end{abstract}

Keywords - Chaotic dynamics, chaos, bifurcation, electronic circuits, engineering applications.

Resumo- O presente artigo apresenta estudos numéricos e experimentais, evidências de bifurcação e caos em sistemas eletrônicos, por meio de análises numéricas e experimentais. Bifurcação e caos são constatados em circuitos eletrônicos muito simples, como um circuito $R L D$ (Resistor-Indutor-Diodo), um sistema eletrônico que emula o sistema de Duffing e um conversor eletrônico CC-CC Buck. Para o conversor CC-CC Buck, verificou-se a presença de comportamento caótico por meio de análise numérica e experimental, sendo as mesmas equivalentes.

Palavras-chave - Dinâmica caótica, caos, bifurcação, circuitos eletrônicos, aplicações em engenharia.

\section{Introdução}

Dinâmica não linear é o estudo do comportamento de sistemas cujas equações de evolução no tempo são não lineares, ou seja, as variáveis dinâmicas que descrevem as propriedades do sistema aparecem nas equações matemáticas que descrevem o sistema na forma não linear.

Há na literatura diferentes modelos, teóricos e experimentais, para estudar e caracterizar o comportamento de sistemas dinâmicos não lineares. Monteiro (2006) e Ferrara e Prada (1994) apresentam em seus livros uma série de exemplos com ilustrações e detalhadas análises, além de um extensa lista de referências.

Uma classe de modelos que tem sido importante para o estudo de sistemas dinâmicos são os circuitos eletrônicos. Os circuitos eletrônicos caóticos vêm se firmando como ambientes ideais para a exploração experimental de alguns fenômenos típicos da dinâmica não linear e caótica (Rocha et al., 2005; Sprott, 2000).

Dois circuitos tradicionalmente explorados são o circuito de Chua (Chua, 1994; Chua, 1987) e o oscilador de Colpitts (Kennedy, 1994). Segundo Turci (2005), são circuitos de "construção elaborada". Diante desse contexto e do ponto de vista prático-didático para ensino e estudo do comportamento caótico, espera-se poder trabalhar com circuitos eletrônicos tão simples quanto possível, de modo que os mesmos apresentem características de caos.
Neste trabalho estudou-se três tipos de circuitos eletrônicos, bem como sua implementação por meio de experimentos de simulação e empíricos. Desse modo, caracterizou-se o comportamento caótico em sistemas eletrônicos simples, e de fácil construção (para o caso empírico) e simulação.

Dentre os circuitos eletrônicos, utilizou-se um circuito $R L D$, constituído de um indutor, um diodo, uma resistência e uma fonte senoidal (Hanias et al., 2009), um circuito eletrônico analógico que emula o comportamento dinâmico de sistemas físicos e matemáticos, em particular o sistema de Duffing (Battelli e Palmer, 1993), e, em especial, um conversor eletrônico de potência CC-CC Buck.

Circuitos eletrônicos chaveados apresentam uma dinâmica "rica". Uma das características desses circuitos é a operação comutada entre diferentes topologias de circuitos, o que ocasiona uma variedade de comportamentos não lineares, incluindo a ocorrência de cenários específicos de bifurcações e a presença de evolução caótica (Turci, 2009). Há uma série de trabalhos preocupados em descrever o comportamento caótico dos conversores (Giaouris et al., 2012; Taborda et al., 2012; Tse, 1994b; Deane e Hamill, 1990).

Estes circuitos são fáceis de construir, exibem uma variedade de comportamentos dinâmicos e oferecem uma excelente oportunidade para uma comparação detalhada com a teoria. Desse modo, o principal objetivo do artigo é constatar fenômenos não lineares e comportamentos caóticos em 
circuitos eletrônicos de simples construção, simulação e implementação, do ponto de vista numérico (por meio de simulações) e prático (conversor CC-CC Buck).

O presente artigo está organizado como segue: a presente seção introduziu o tema a ser estudado, bem como apresentou o presente trabalho. A seção 2 apresenta a descrição e configuração dos circuitos eletrônicos analisados de forma numérica (circuito $R L D$ e Duffing) e experimental (conversor CC-CC Buck). A seção 3 apresenta os resultados obtidos pela análise numérica de simulações, mostrando a presença de caos em circuitos eletrônicos muito simples. Após, na seção 4, são apresentados e discutidos os resultados experimentais obtidos na implementação do conversor CC-CC Buck. Por fim, são apresentadas as considerações finais e agradecimentos.

\section{Descrição e configuração dos circuitos}

\subsection{Circuito $R L D$}

Considere um circuito constituído de um diodo $(D)$, um indutor $(L)$ e uma resistência $(R)$, excitado por um sinal senoidal $\left(V_{i n}\right)$, como mostra a Figura 1, sendo o diodo o elemento não linear do circuito. Para tensões mais elevadas, a capacitância interna do diodo depende fortemente da tensão sobre o elemento, deixando de ser constante.

A Equação 1 mostra o sistema de equações diferenciais, construídas a partir da análise das tensões de Kirchhoff. A tensão no diodo é dado pela expressão entre parênteses. $V_{d}$ consiste em um modelo de capacitâncias linear por partes com uma pequena tensão de offset $\left(E_{O}\right) . C_{j}$ e $C_{d}$ são, respectivamente, capacitâncias de depleção e difusão do diodo. Detalhes da descrição do circuito podem ser vistos em Turci (2009) e Hanias et al. (2009). Nesse sistema, o parâmetro de bifurcação é a amplitude do sinal de entrada.

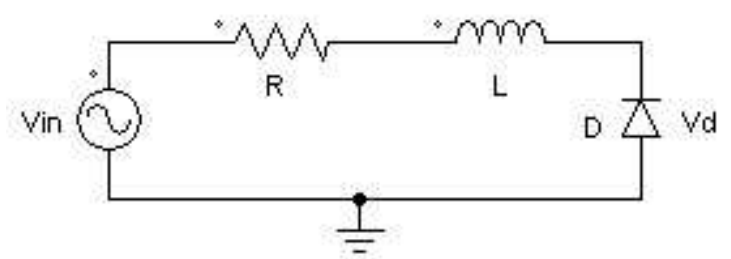

Figura 1: Circuito a diodo. $V_{d}$ queda de tensão no diodo D. 
é mantida através do diodo (roda livre). O indutor descarrega auxiliando o capacitor a manter a energia na carga; e iii) chave ainda aberta, o indutor é totalmente descarregado, o diodo bloqueia e somente o capacitor mantém a tensão na carga.

O fato de operar em regime descontínuo, aliado ao circuito realimentado, propicia o aparecimento de comportamento caótico no conversor.

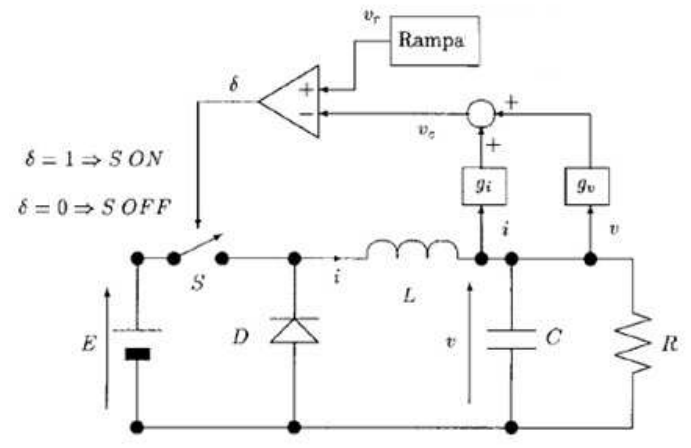

Figura 3: Conversor Buck realimentado (Bernardo et al., 1998).

O modelo dinâmico para o conversor é apresentado em (3). Quando a tensão de saída é menor que a tensão de referência a chave é fechada $(\delta=1)$ e quando a tensão de saída é maior que a tensão de referência a chave é aberta $(\delta=0)$ (Bernardo et al., 1998; Tse, 1994a). 


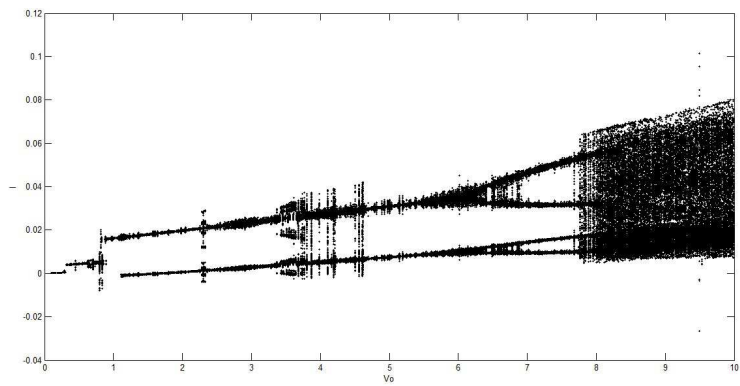

Figura 7: Circuito RLD. Diagrama de bifurcação. $V_{o}$ parâmetro de controle.

Contudo, por meio da Figura 10 é possível visualizar algumas transições.
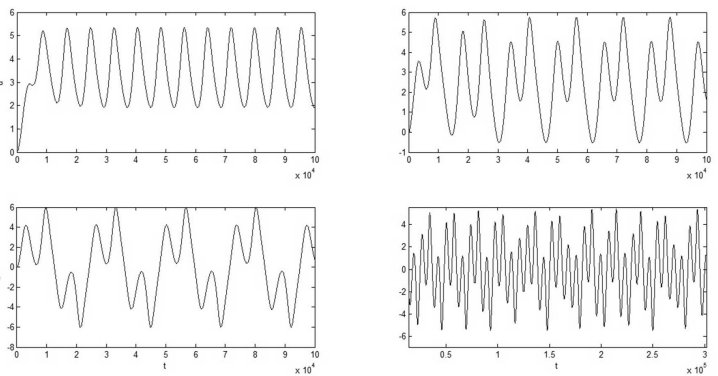

Figura 8: Duffing.Forma de onda temporal. Períodos $1,2,3$ e caos.
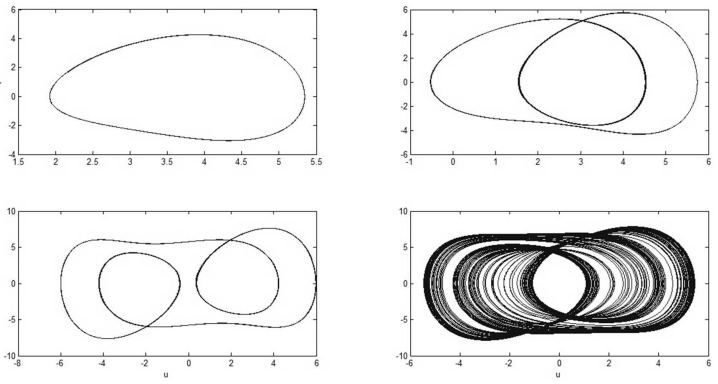

Figura 9: Duffing. Atratores. Períodos 1, 2, 3 e caos.
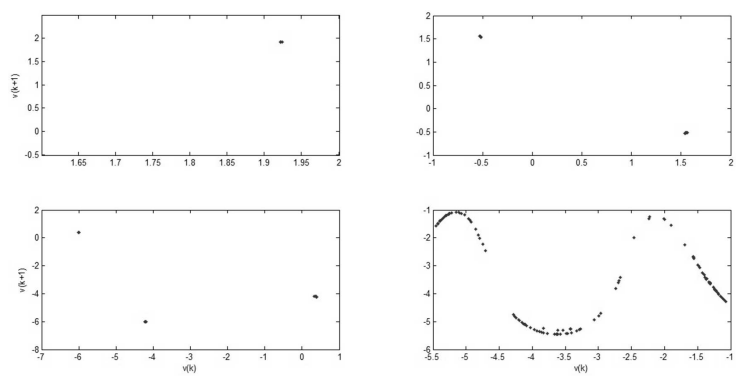

Figura 10: Duffing. Mapa de Poincaré. Períodos $1,2,3$ e caos.

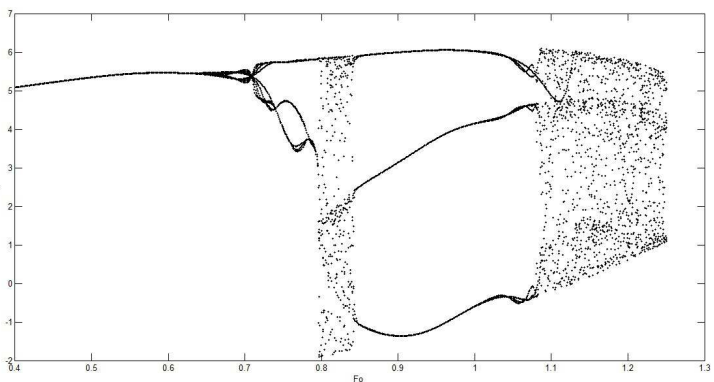

Figura 11: Duffing. Diagrama de bifurcação. $F_{o}$ parâmetro de controle.

\subsection{Conversor $C C-C C$ Buck}

A Figura 12 mostra o circuito implementado no PSIM ${ }^{1}$. O mesmo circuito foi implementado em laboratório, como será apresentado na próxima seção.
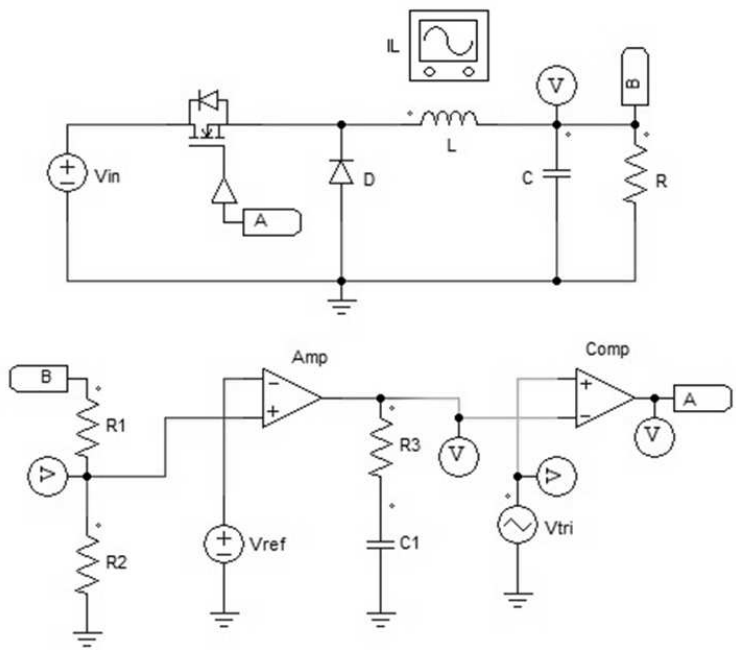

Figura 12: Circuito do conversor Buck. Circuito prático utilizado tanto para simulação quanto para a montagem experimental.

a) Contínuo (ver Equação 3)

Realizou-se a simulação do conversor Buck para duas situações: período 1 e regime caótico. A Figura 13 ilustra os atratores e a evolução temporal para as duas situações.

\section{b) Discreto (ver Equação 4)}

O diagrama de bifurcação do conversor, mostrado na Figura 14 apresenta uma rota para o caos por dobramento de período. O número de Feigenbaum (ver Equação 5), que foi proposto como uma característica universal de razões entre os valores dos parâmetros de controle em cada uma das bifurcações na rota para o caos, é bem definido no 

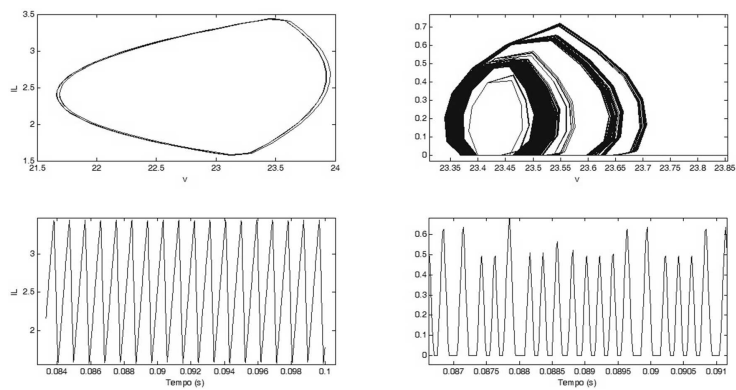

Figura 13: Conversor Buck. Atratores, corrente no indutor (eixo $\mathrm{x}$ ) e tensão de saída (eixo y) e (abaixo) formas de onda temporal da corrente no indutor. Período 1 e caos. Figura ampliada.

diagrama do conversor. Para os dados simulados apresentou um valor aproximado de 4,2 .

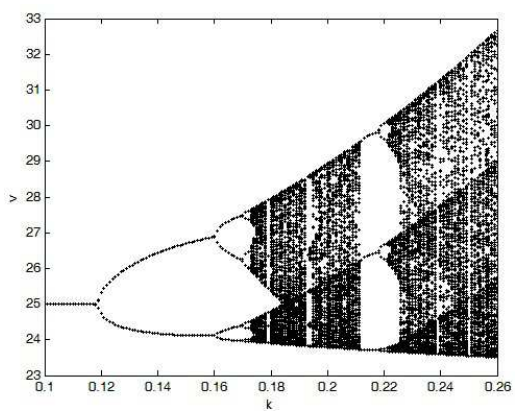

Figura 14: Diagrama de bifurcação para o conversor Buck. Parâmetro de bifurcação $k$, constante relacionado ao ganho de realimentação.

$$
\sigma_{\lim _{n \rightarrow \infty}}=\underline{\alpha_{n}-\alpha_{n-1}}
$$



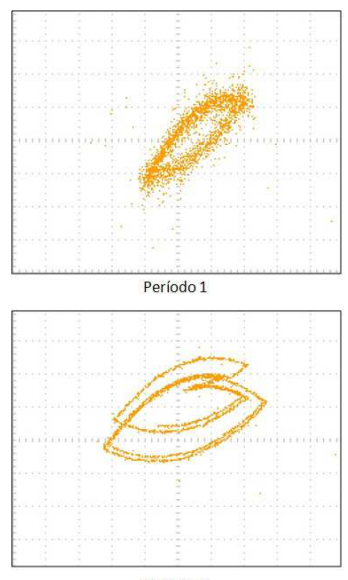

Período 4
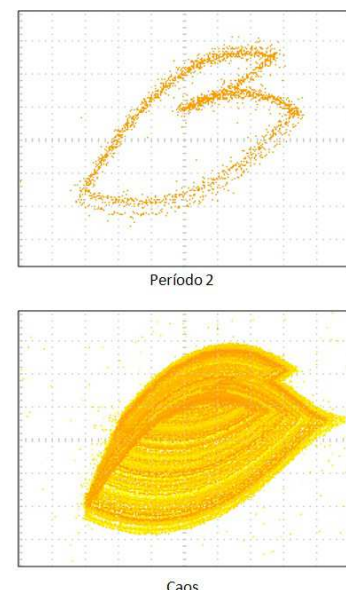

Caos
Figura 17: Atrator, corrente no indutor (eixo x) e tensão de saída (eixo y). Conversor Buck. Períodos $1,2,4$ e caos.

\section{Agradecimentos}

Agradecimentos ao $\mathrm{CNPq}$ e à agência de fomento CAPES - Brasil, pelo apoio financeiro para realização do presente trabalho. Ao CEFET-MG, que disponibilizou os laboratórios para ensaio e testes experimentais.

\section{Referências}

Battelli, F. e Palmer, K. J. (1993). Chaos in the Duffing equation, Journal of Diferential Equations 101(2): 276-301. DOI: $10.1006 /$ jdeq. 1993.1013

Bernardo, M., Garofalo, F. Glielmo, L. e Vasca, F. (1998). Switchings, bifurcations, and chaos in DC-DC converters, IEEE Transacions on Circuits and Systems - I: Fundamental Theory and Applications 45(2): 133-141.

Chua, L. O. (1987). Special issue on chaotic systems, Proc. IEEE 75(8): 979-1120. DOI: 10.1109/PROC.1987.13844

Chua, L. O. (1994). Chua circuit - an overview 10 years later, Journal of Circuits Systems and Computers 4(2): 117-159. DOI:

10.1142/S0218126694000090

Deane, J. H. B. e Hamill, D. C. (1990). Analysis, simulation and experimental study of chaos in the buck converter, Power Electronics Specialists, pp. 491-498.

Ferrara, F. N. e Prada, C. P. C. (1994). Caos uma introdução, 1 edn, Edgar Blucher.

Fradkov, A. L. e Evans, R. J. (2005). Control of chaos: Methods and applications in engineering, Annual Reviews in Control 29: 33-56. DOI: 10.1016/j.arcontrol.2005.01.001

Giaouris, D., Stergiopoulos, F., Ziogou, C. e Ipsakis, D. (2012). Nonlinear stability analysis and a new design methodology for a
PEM fuel cell fed DC-DC boost converter, International Journal of Hydrogen Energy 37(23): 18205-18215. DOI:

10.1016/j.ijhydene.2012.09.002

Hanias, M. P., Avgerinos, Z. e Tombras, G. S. (2009). Period doubling, feigenbaum constant and time series prediction in an experimental chaotic RLD circuit, Chaos, Solitons e Fractals 40: 1050-1059. DOI: 10.1016/j.chaos.2007.08.061

Kennedy, M. P. (1994). Chaos in the Colpitts oscillator, IEEETransactions on Circuits and Systems I-Fundamental Theory and Applications 41(11): 771-774. DOI: $10.1109 / 81.331536$

Monteiro, L. H. A. (2006). Sistemas Dinâmicos, 2 edn, Livraria da Física.

Rocha, R., Filho, L. S. M. e Machada, R. F. (2005). Analogia eletrônica no ensino de física, Revista Brasileira de Ensino de Fı'sica 27(2): 211-218. DOI: 10.1590/S0102-47442005000200005

Santos Filho, R. M., Pinto, M. A. S. e Souza, J. K. S. (2003). Low cost embedded kits for teaching power electronics in laboratory, VII COBEP - Congresso Brasileiro de Eletrônica de Potência.

Savi, M. A. (2004). Dinâmica não-linear e caos. Notas de aula - COPPE - Universidade Federal do Rio de Janeiro.

Sprott, J. C. (2000). A new class of chaotic circuit, Physics Letters A 266: 19-23. DOI: 10.1016/S0375-9601(00)00026-8

Taborda, J. A., Angulo, F. e Olivar, G. (2012). Characterization of chaotic attractors inside band-merging scenario in a zad-controlled buck converter, International Journal of Bifurcation and Chaos 22(10): 1-34. DOI: 10.1142/S0218127412300340

Tse, C. K. (1994a). Chaos from a buck switching regulator operating in discontinuous mode, International Journal of Circuit Theory and Applications 22: 263-278. DOI: $10.1002 /$ cta. 4490220403

Tse, C. K. (1994b). Flip bifurcation and chaos in three-state boost switching regulators, IEEE Transacions on Circuits and Systems - I: Fundamental Theory and Applications 41(1): 16-23.

Turci, L. F. R. (2005). Caracterização e controle de caos em circuitos chaveados, Dissertação, Instituto Tecnológico de Aeronáutica.

Turci, L. F. R. (2009). Caracterização, controle de caos e sincronização em circuitos chaveados, Tese, Instituto Tecnológico de Aeronáutica. 